\title{
Comparison of Measured and Simulated Structural Loads of an Offshore Wind Turbine at Alpha Ventus
}

\author{
Kolja Müller, Mario Reiber and Po Wen Cheng \\ Stuttgart Wind Energy, Institute of Aircraft Design, University of Stuttgart \\ Stuttgart, Baden Wurttemberg, Germany
}

\begin{abstract}
A comparison of fatigue and extreme loads from simulations with full-scale measurements collected over a period of ten months in the offshore test field, Alpha Ventus, is presented in this paper. There are two goals of this study: (1) to check if the measured range of fatigue and extreme loads can be captured correctly by simulations when the variations of relevant environmental parameters are taken into account; and (2) to investigate if measured extreme loads can be reproduced by simulations when ten-minute averages of the environmental parameters are used. The results show a good overall match of loads when the variation of environmental parameters is considered but an insufficient match when the events of maximum load occurrence are compared.
\end{abstract}

\section{INTRODUCTION}

The site-specific design of offshore wind turbines requires the use of simplified assumptions of the environment in order to limit the number and detail of simulations to be performed. Additionally, a set of physical assumptions is implied in the various aeroservo-hydro-elastic models used for the simulation of the loads of offshore wind turbines. These include models for the wind and wave environment and models for the load transfer from the environment to the turbine and between system components.

The use of simplified environmental assumptions is generally justified by the use of conservative estimates for environmental parameters (Türk and Emeis, 2010). The verification and validation of the models used to describe offshore wind turbines involve code-to-code comparisons (Jonkman and Musial, 2010; Popko et al., 2012) and comparisons to scaled experimental data (Müller et al., 2014).

To complete the design process and learn from it, a thorough validation of physical models at full scale and subsequent environmental assumptions are necessary in order to identify shortcomings and highlight the potential for less conservative designs and/or additional simplifications within the process of site-specific project certification.

Söker et al. (2006) presented a procedure for full-scale load validation for onshore wind turbines. Guidance for load validation can also be taken from IEC TS 61400-13 (2001). Generally, the goal is to validate the models on the basis of specific environmental events, which has been done in previous studies (Yde et al., 2015; Zierath et al., 2014; Koukoura et al., 2013; Kaufer and Cheng, 2014). Regarding offshore wind turbines, a simplified set of load cases for load validation at full scale is presented:

(1) Gravity load case: Without environmental influence, turbine in parked or idling position, for sensor calibration.

(2) Nacelle or rotor revolution: Without environmental influence, for sensor calibration.

(3) Frequency domain comparison: Moderate environmental conditions (below rated wind speed, low turbulence intensity, and low wave height)

Received December 18, 2015; revised manuscript received by the editors April 11, 2016. The original version was submitted directly to the Journal.

KEY WORDS: Offshore, wind energy, simulation, measurements, alpha ventus, design of experiment, certification.
(4) Statistical load comparison: Average environmental data compares the minimum, maximum, mean, and standard deviation of loads.

As part of the University of Stuttgart-led RAVE project, OWEA LOADS, the validity of simulation models and design procedures is investigated. Data is made available from the offshore test field, Alpha Ventus (DOTI, 2015), which provides a unique opportunity to validate both numerical models and environmental assumptions due to the extensive data of both turbine loads and simultaneous environmental conditions. Load measurements from the Senvion $5 \mathrm{M}$ turbine fixed on a jacket substructure are used along with the environmental measurements from the FINO1 platform (see Fig. 1).

For the considered turbine, Kaufer and Cheng (2014) carried out a full-scale validation of the simulation model describing the considered turbine and substructure on the basis of specific environmental conditions. On the basis of the full-scale measurements, state-of-the-art load validation was performed, and it was shown that the integrated simulation models for offshore wind turbines are able to capture relevant loads if the environmental conditions are comparable for both simulations and measurements (see load case 4 above).

In an overall validation procedure of the site-specific design of offshore wind turbines, the subsequent step in the model val-

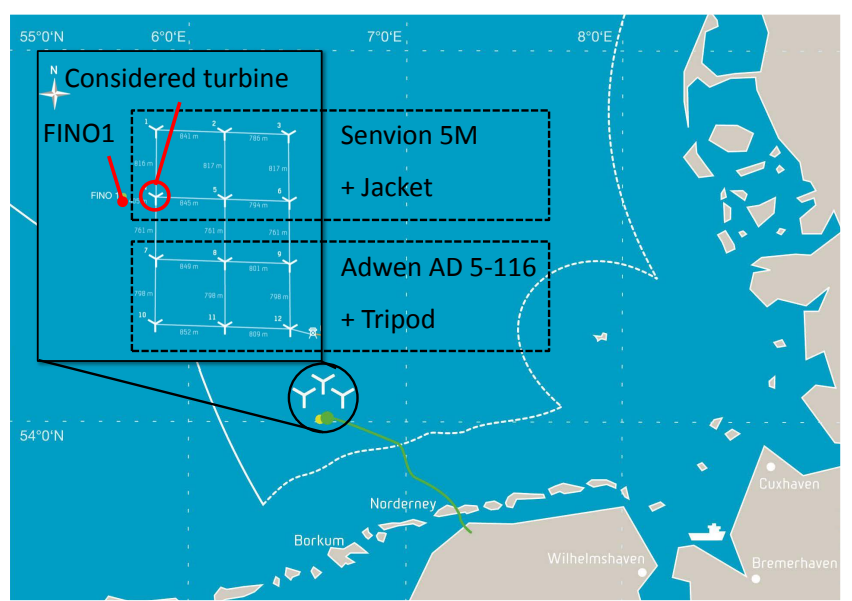

Fig. 1 Location and layout of Alpha Ventus (DOTI, 2015) 
idation is the validation of environmental assumptions that are applied in the design process. However, a simple comparison of simulated with measured loads shows that loads observed from measured data exhibit a large range that cannot be captured when the assumptions provided in guidelines are applied. This results from the limited consideration of the variation of environmental parameters, e.g., only wind speed and wind direction are included in the Design Load Case (DLC) 1.2 description of fatigue assessment (IEC TS 61400-3, 2009). A few of the underlying assumptions for DLC 1.2 are a uniform wind shear for all relevant design situations and a deterministic dependence of the turbulence, wave height, and wave period on the wind speed.

Building on the validated simulation model and preparing for the validation of environmental assumptions, this study aims at investigating the capability of the previously applied model to predict load variation that is experienced in the environment and deemed to originate from the variation of environmental conditions. Regarding extreme loads in particular, in addition to an investigation of the load variation, focus needs to be placed on the very largest of loads experienced in the environment. The applied physical models should be able to reproduce the largest loads. This is considered to be ambitious as the highest measured extreme loads are likely to be caused by transient events that are not captured by the available measurements used for simulation model input, i.e., typically ten-minute mean data. However, as environmental models do take into account the stochastic behavior of the environment, it can be possible to obtain comparable loads from simulation models when mean data are considered.

Summarizing the above, we address two questions in this study:

(1) Can the measured range of the fatigue and extreme loads of an offshore wind turbine be adequately captured by simulations when the observed variation of environmental parameters is considered?

(2) Is it possible to reproduce the largest measured extreme loads through simulations based on available measurements of environmental parameters?

Both questions are answered by two independent simulation studies. The focus lies on the assumptions of the IEC 61400-3 (IEC TS 61400-3, 2009) power production design load cases 1.1 (extreme loads during normal power production) and 1.2 (fatigue loads during normal power production). Numerous measurements of these cover loads during normal operating conditions of the turbine are available, and thus these cover loads present a good basis for the validation at full scale.

The first study aims at reproducing the range of fatigue and extreme loads measured at the investigated turbine on the test wind farm. In order to achieve this, five environmental parameters are varied across three different operating conditions of the turbine. This leads to a significant amount of simulations that needs to be performed. Thus, in the course of the study, so-called design of experiment (DOE) methods are evaluated for applicability to this kind of simulation study. DOE methods allow for simulations with a reduced number of load cases and thus can reduce the simulation effort of this kind of study. The results of the DOE study also enable an extensive sensitivity analysis at various points along the offshore wind turbine structure, which is presented for fatigue loads. The range of extreme loads from this study is compared to measured extreme loads together with the results of the second study.

The second study aims at reproducing the largest extreme loads that are measured during the same period of time. Here, the maximum loads are identified, and correlating environmental conditions are used as simulation input. The results are then compared to the measurements, and a check is performed if the environmental conditions fed into the simulation results in extreme loads as well.

\section{REFERENCE WIND TURBINE AND MEASUREMENTS}

The presented work uses measurement data from a Senvion $5 \mathrm{M}$ turbine that are available from the German offshore test field, Alpha Ventus, through the RAVE project, OWEA Loads (OWEA Loads, 2015). On the wind farm, two different turbine types (Adwen and Senvion) with different substructures (tripod and jacket) are equipped with sensors measuring environmental conditions as well as loads and accelerations. Additionally, further environmental data are available from the metmast FINO1 (FINO-Offshore, 2015) located about $400 \mathrm{~m}$ west of the analyzed turbine (see Fig. 1). FINO1 environmental data are freely available to research projects.

The present study focuses on the wind turbine of the Senvion $5 \mathrm{M}$ type with $5 \mathrm{MW}$ rated power (see Fig. 2). The cut-in wind speed is $v_{\text {cutin }}=3.5 \mathrm{~m} / \mathrm{s}$, the rated wind speed is $v_{\text {rated }}=13 \mathrm{~m} / \mathrm{s}$, and the cut-out wind speed is $v_{\text {cutout }}=30 \mathrm{~m} / \mathrm{s}$ (DOTI, 2015). The turbine has a hub height of $92 \mathrm{~m}$ with a rotor diameter of $126 \mathrm{~m}$ and is positioned on a jacket substructure, which results in a hub height of $120 \mathrm{~m}$ with reference to the mudline. Four pairs of $\mathrm{x}$ shaped braces stiffen the jacket legs. The water depth at the site is about $28 \mathrm{~m}$ with tides present.

Data from over 100 sensors at multiple positions on the rotornacelle assembly (RNA), tower, and jacket above and below sea level are available. These sensors record environmental conditions such as the wind speed and wind direction as well as corrosion, loads, accelerations, and the turbine status (i.e., supervisory control and data acquisition (SCADA) data). In particular, each position on the turbine and the substructure is equipped with up to four sensors in order to capture the motion, stresses, and moments that are calculated from the stress sensors. The data collection started at the beginning of 2011 and is still ongoing although many of the sensors have experienced defects or show significant variation over time that has made them difficult to use (e.g., the slope and offset as well as the offset drift). Data collection, calibration, and plausibility checks for the Senvion $5 \mathrm{M}$ data are performed by the

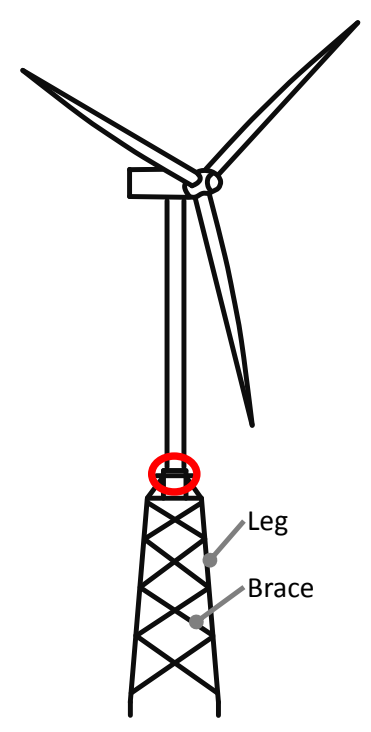

Fig. 2 Sketch of Senvion 5M with jacket substructure. Position of measurements for evaluations is highlighted in red. 
project partners, and the data is uploaded afterwards to a server that is accessible online by research partners. For this work, errors and uncertainties included in the measurements are not regarded but could have an additional influence on the uncertainty of the measurements.

Statistical data and high-resolution $(50-\mathrm{Hz})$ data for time periods of ten minutes are available and are used in the presented work. The focus lies on the tower base fore-aft bending moment as the corresponding data from strain gauges installed in a full-bridge configuration show good quality and availability on the basis of the performed plausibility checks. Four strain gauges positioned around the tower base are combined into two bending moments. Together with the information on the azimuth angle of the nacelle, these are geometrically combined to provide the fore-aft bending moment. Measurements taken over the course of ten months between April 2011 and January 2012 are analyzed. An investigation of further sensors has shown that the behavior of the tower base fore-aft bending moment is qualitatively similar to that of the side-side bending moment in that the loads in the jacket legs are dependent on the wind speed; therefore, the side-side bending moment and its loads are not evaluated in this study.

\section{Selection of Measurements}

An initial data screening shows widespread load data that are not feasible for effective load validation (see Fig 3). In order to focus on relevant and useable data during this study, four limiting steps are applied to the available data regarding (1) the inflow condition; (2) the turbine status; (3) the time period related to the sensor availability and long-term turbine status; and (4) the treatment of outliers. Firstly, only free flow conditions are considered. This means that the data are neglected when the wind turbine is in the wake of another wind turbine and the anemometers at FINO1 are shadowed by the met mast structure. (The wind data is obtained from the FINO1 met mast.) (Westerhellweg et al., 2011). Secondly, only power production conditions without faults are taken into account in order to comply with the International Electrotechnical Commission (IEC) power production load cases (IEC TS 61400-3, 2009). Thirdly, a time period of almost ten months is considered between April 2011 and February 2012. During this time period, the quality of the data is high, and the turbine is not curtailed in power that would lead to lower thrust forces above the rated wind speed. Various sensors focusing on both environmental and load conditions are part of the investigation and thus need to provide reliable data throughout the considered time period. Fourthly, an analysis of outliers in damage equivalent loads (DELs) leads to the conclusion that transient events with a high variation of power output (e.g., start-up or shut-down procedures) are very likely to cause unusual high load amplitudes and consequently influence the associated DEL. As these transient events are not considered in the power production load cases in certification guidelines, only measurements showing the DEL within a range of \pm 2 . the standard deviation of the scatter within a wind bin (with bin size $=1 \mathrm{~m} / \mathrm{s}$ ) are considered. Note that even though it is not directly linked to extreme loads, this limitation is also used for the extreme load evaluation as the same events (start-up or shut-down) are not included in the DLC 1.1 description as well.

Figure 3 shows the final selection of the measurement data. The figure suggests that higher fatigue loads originate from wind directions other than the free flow (e.g., wake conditions), which can be linked to added wake turbulence. The effect of added wake turbulence is not considered here, and it is noted that this study, for simplicity, only considers a subset of the loads that are to be expected in the real environment.

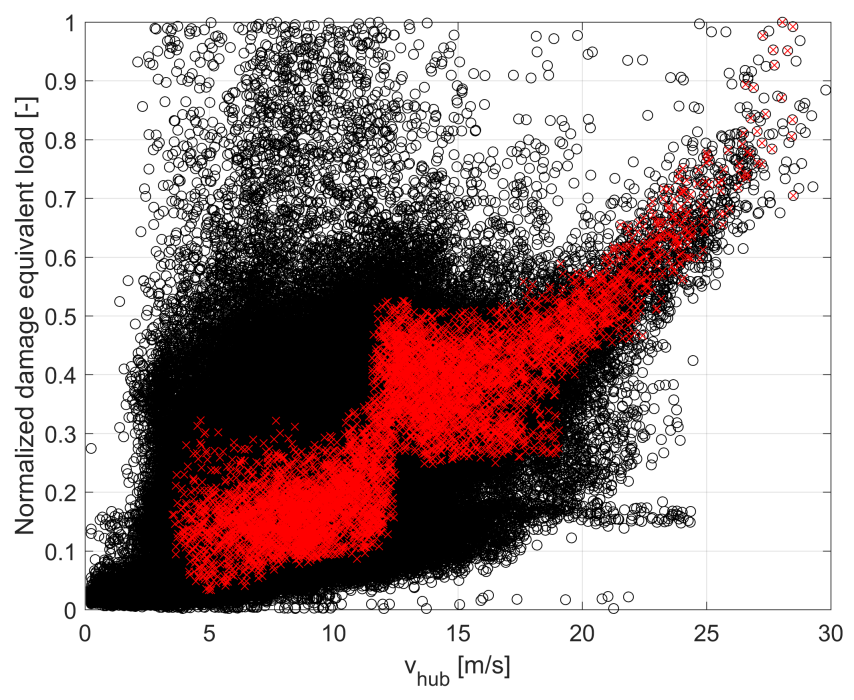

Fig. 3 Damage equivalent loads (DELs) of measured tower base fore-aft bending moment showing both the complete (black) and selected dataset (red) for the presented study

\section{APPLIED SIMULATION MODEL}

The applied wind turbine model is set up in the coupled simulation environment Flex5-Poseidon. This was developed in collaboration with the Leibniz University Hannover within the RAVE project (RAVE, 2015). It combines the aero-servo-elastic simulation tool Flex5 (Øye, 1999) with the linear finite element method (FEM) tool Poseidon for space frame structures affected by wave loads (Böker, 2010). The simulation environment has previously been verified (Popko et al., 2012) and validated in the time and frequency domain and by statistical data (Kaufer and Cheng, 2014). The controller and relevant parameters of the wind turbine are provided by Senvion $\mathrm{GmbH}$, and the data regarding the jacket substructure are provided by OWEC Tower AS (Owec Tower AS, 2015).

\section{Simulation Settings}

On the aerodynamic side, individual wind fields are generated for each simulation through the use of VindSim, the turbulence simulator of Flex5. The Kaimal turbulence model is applied, and six seeds for each combination of environmental parameters are considered with varying yaw inflow angle, i.e., $-8^{\circ}, 0^{\circ},+8^{\circ}$, in order to account for statistical variance.

With respect to the hydrodynamics, buoyancy effects are considered, and the legs of the jacket are flooded while the braces are filled with air. Interaction with the soil is modeled through the use of equivalent beam elements. Marine growth is assumed to be $0.05 \mathrm{~m}$ in thickness. The Joint North Sea Wave Project (JONSWAP) wave spectrum is used for stochastic sea state generation. The transition piece, which originally consists of concrete, is also modeled with beam elements that show equivalent dynamic properties.

The simulation length is set to 660 seconds with a time step of 0.02 seconds. The first 60 seconds of each simulation are cut off in order to eliminate transient effects at the beginning of the simulation. For each design point for the six simulations, the mean value of the DEL is used for the evaluation of fatigue loads. For the extreme loads, maximum values are obtained from the simulation results, and the mean and maximum of the maximum values are compared to the measurements. 


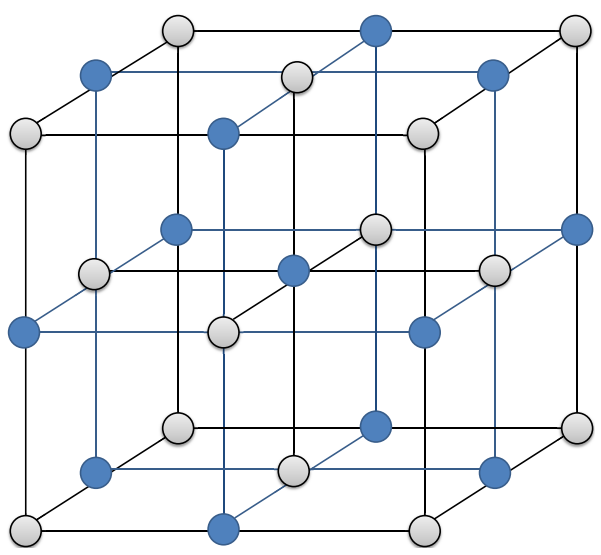

Fig. 4 Exemplary presentation of a three-factor, full factorial design with three levels of variance (all points) and the corresponding Box-Behnken design (only blue/dark points). The visualized points represent design points (the combination of considered factor levels) of experiments that need to be performed.

\section{BOX-BEHNKEN DESIGN FOR CONSIDERATION OF VARIATION OF ENVIRONMENTAL PARAMETERS}

The design of experiments is a methodology used traditionally for the planning of physical or qualitative experiments. When simulation studies are performed, a number of variables or factors are varied. If thorough information on the behavior of a system is sought, the simplest simulation setup is to consider a so-called full factorial design (see Fig. 4). Through regression analysis, the results can then be transformed into a meta-model or response surface. The order of this model depends on the number of levels applied in the study, i.e., how many points are simulated for each considered variable. In the presented work, a factor level of three is chosen so that the minimum, mean, and maximum values of each environmental variable are considered. With an increasing number of variables, however, full factorial designs become less feasible. The general goal of the design of the experiments is to organize experimental procedures efficiently so that the necessary level of detail of information is attained through the use of a minimum number of experimental runs (Gundlach, 2004; Kleijnen, 2008; Montgomery, 2013). If the setup of the design of the experiment is performed through the use of quantitative factors, response surfaces or meta-models of the simulation model can be determined efficiently. The resulting meta-models can then be used to provide results with respect to a considered set of input variables.

As the design of offshore wind turbines requires the consideration of numerous environmental parameters, the design of experimental methods can support a more precise and thus less conservative design. In particular, this is the case for a fatigue load evaluation where the reference value is the accumulated damage over a lifetime.

One popular method for the determination of meta-models, which is applied in the present study, is the Box-Behnken design (Box and Behnken, 1960). To exemplify the definition of design points through the use of the design of experimental methods and to show the application of the Box-Behnken design, a threefactor, full factorial design with three factor levels can be used (Gundlach, 2004). The design points of the Box-Behnken design are defined by four spheres created around the center point of a cube that is created by the considered factor ranges. The zeroth sphere is the central point itself, the first sphere tangents the centers of the surfaces of the cube faces, the second sphere tangents the centers of the edges, and the third sphere crosses the corners of the square. The Box-Behnken design now limits the number of design points by neglecting selected spheres. The previous example can be applied in an analogous way to higher dimensions. A hypercube is thus created with Box-Behnken design points.

The definition of the Box-Behnken design ensures the separation of combined factor effects (orthogonality), links the variance of the predicted response values closely to the distance from the center of the considered hypercube (rotatability), and provides a simple setup and evaluation of the response values. The BoxBehnken design also has a low factor of redundancy $R=N / L$, where $N$ is the number of design points and $L$ is the necessary coefficients to describe the meta-model. For the present study, the redundancy factor is $R<2$ compared to $R>10$ for a full factorial design.

If the Box-Behnken design is applied with three factor levels, a meta-model of the second order can be derived:

$y=b_{0}+\sum_{i=1}^{k} b_{i} x_{i}+\sum_{i=1}^{k} \sum_{j=i}^{k} b_{i j} x_{i} x_{j}$

where $y$ is the modeled value (in this work the DEL), $b_{i}$ are the regression coefficients, $x$ are the varied factors (in this work the environmental parameters) (see Table 1$), i, j$ are the iteration coefficients, and $k$ is the number of varied factors (in this work $k=5$ ).

The regression coefficients $b_{0}, b_{i}, b_{i j}$, and $b_{i i}$ of a Box-Behnken meta-model based on five factors are calculated as follows (Box and Behnken, 1960):

$b_{0}=\bar{y}_{0}$

$b_{i}=\frac{1}{16} \cdot \sum_{u=1}^{N} x_{i u} y_{u}$

$b_{i j}=\frac{1}{4} \cdot \sum_{u=1}^{N} x_{i u} x_{j u} y_{u}, \quad i \neq j$

$b_{i i}=\frac{1}{12} \cdot \sum_{u=1}^{N} x_{i u}^{2} y_{u}-\frac{1}{96} \sum_{u=1}^{N} x_{j u}^{2} y_{u}-\frac{\bar{y}_{0}}{2}$

\begin{tabular}{cccccr}
\hline & $\begin{array}{c}v_{\text {hub }} \\
{[\mathrm{m} / \mathrm{s}]}\end{array}$ & $\begin{array}{c}T i \\
{[-]}\end{array}$ & $\begin{array}{c}H_{s} \\
{[\mathrm{~m}]}\end{array}$ & $\begin{array}{c}T_{p} \\
{[\mathrm{~s}]}\end{array}$ & $\begin{array}{c}\alpha \\
{[-]}\end{array}$ \\
\hline PLR & & & & & \\
- & 4.0 & 0.043 & 0.13 & 2.5 & -0.08 \\
0 & 7.5 & 0.053 & 1.38 & 7.0 & 0.14 \\
+ & 11.0 & 0.063 & 2.63 & 11.5 & 0.38 \\
TLR & & & & & \\
- & 11.0 & 0.043 & 0.63 & 2.5 & 0.02 \\
0 & 13.0 & 0.047 & 2.00 & 7.0 & 0.19 \\
+ & 15.0 & 0.051 & 3.38 & 11.5 & 0.36 \\
FLR & & & & & \\
- & 15.0 & 0.050 & 0.63 & 2.5 & 0.02 \\
0 & 22.5 & 0.058 & 3.75 & 7.0 & 0.08 \\
+ & 30.0 & 0.066 & 6.88 & 11.5 & 0.14 \\
\hline
\end{tabular}

$v_{h u b}$ is the hub height wind speed; $T i$ is the turbulence intensity; $H_{s}$ is the significant wave height; $T_{p}$ is the wave peak period; $\alpha$ is the wind shear exponent; PLR is the Partial Load Range; TLR is the Transition Load Range; and FLR is the Full Load Range.

Table 1 Chosen variation of environmental parameters in presented study 
where $\bar{y}_{0}$ is the mean value of all results in the center points, $u$ is the row index of the experiment matrix, $N$ is the number of rows, $i$ is the column index of the design table, and $y_{u}$ is the result of the $u$-th design point. $x_{i}$ represents a "dummy" variable that indicates the level of the considered factor, i.e., the min, mean, and max values.

In the present study, compared to a full factorial design with 4,374 simulations, only 738 simulations $(<17 \%)$ are necessary when the Box-Behnken methodology is used for the selection of design points.

\section{SELECTION AND DEFINITION OF LOAD CASES}

The definition of both the full factorial and the Box-Behnken design point selection is described in this section. The results (DEL and extreme loads) are compared to the measurement data in order to check if the measured load ranges can be reproduced when a realistic variation of important environmental parameters is taken into account.

\section{Design Point Selection for Load Simulations}

In order to test the applicability of the Box-Behnken method, a full factorial simulation study is performed as a reference. From the results, Box-Behnken design points are selected in the postprocessing stage so that the results of both can be compared to each other and to the measurements.

For the full factorial design, three wind turbine load ranges are defined on the basis of the evaluation of the measured loads (see Fig. 3). The ranges are closely linked to the controller status of the turbine and thus to the wind speed and are the Partial Load Range (PLR), Transition Load Range (TLR), and Full Load Range (FLR). The definition of the wind speed limits of the different load ranges is based on an examination of the measured load distribution. The observed measured loads also indicate that a polynomial regression function of the second order is necessary to adequately capture the load variation with increasing wind speed. For this reason, a threefactor design is chosen that requires three design points (min, max, and mean) for each of the five considered environmental parameters (hub height wind speed $v_{\text {hub }}$, turbulence intensity $T i$, significant wave height $H_{s}$, wave peak period $T_{p}$, and wind shear exponent $\alpha$ ) within each of the load ranges. For the full factorial design, this leads to $3^{\wedge} 5$ design points $\times 6$ simulations $\times 3$ load ranges $=4,374$ simulations.

The definition of the environmental boundary conditions for the setup of the simulations is given on the basis of available measurements (wind speed), hindcast data available to the project (turbulence intensity, wave height, and wave period), and a previous study of environmental conditions at alpha ventus (wind shear) (Türk, 2008). For all of the parameters, maximum, minimum, and mean values are chosen for each of the previously defined load ranges. The wind direction is set to the free flow direction. Windwave misalignment, currents, and tidal influence are not considered. The applied limits of the environmental conditions for each of the load ranges are presented in Table 1. Regarding wave period ranges, this evaluation resulted in equal values for all considered load ranges (see Table 1).

\section{EVALUATION OF FATIGUE LOAD SIMULATION STUDY AND VALIDATION OF STATISTICAL SPREAD OF FATIGUE LOADS}

\section{Processing of Fatigue Load Data}

For the current study, focus is placed on analyzing loads at the tower base, in particular the fore-aft bending moment. For measurements, the data are obtained from four strain gauges at the tower base and the azimuth angle of the turbine, as described in the reference wind turbine and measurements section. For simulations, the fore-aft bending moment is provided as output directly by Flex 5 .

For the first part of the study that focuses on fatigue loads, high-resolution measurements and time series from simulations are obtained, and the rainflow is counted and transferred to the DEL through the use of the following equation:

$\Delta \sigma_{\mathrm{eqv}}=\left(\sum \frac{\Delta \sigma_{i}^{m} \cdot n_{i}}{N_{\mathrm{ref}}}\right)^{1 / m}$

where $\Delta \sigma_{\text {eqv }}$ is the DEL and $N_{\text {ref }}$ is the reference number of load cycles. The considered cycle ranges $\Delta \sigma_{i}$ and corresponding cycle counts $n_{i}$ are obtained by the application of a rainflow counting algorithm (Niesłony, 2009) on time series data. The reference number of load cycles is set to $N_{\text {ref }}=600$ in order to obtain $1-\mathrm{Hz}$ DEL. The slope of the S-N curve is set to $m=4$ as is common for steel components of offshore wind turbines.

The evaluation of the results of the simulations is done in a two-step procedure. Firstly, sensitivities and regression functions are compared between the Box-Behnken and full factorial design point selection methods in order to verify the feasibility of the BoxBehnken method for the determination of fatigue loads. This is done by analysis of the so-called effect plots. Additionally, errors are calculated and compared between the regression function of the Box-Behnken design and the results of the full factorial design, as described in Gundlach (2004). Secondly, the simulation results are compared to the measurement data in order to determine if the load variation can be correctly captured when the observed spread of the environmental parameters is taken into account.

\section{Sensitivity Analysis of Fatigue Loads}

We performed an analysis of the results based on the simulation study by looking at the load effects for a detailed evaluation of the influence of environmental parameters on different parts of the turbine. Note that this investigation is based only on the simulation results, and no comparison to the measurement data is performed as the available measurement data is limited to the tower base fore-aft bending moment. For this part, statistical values are

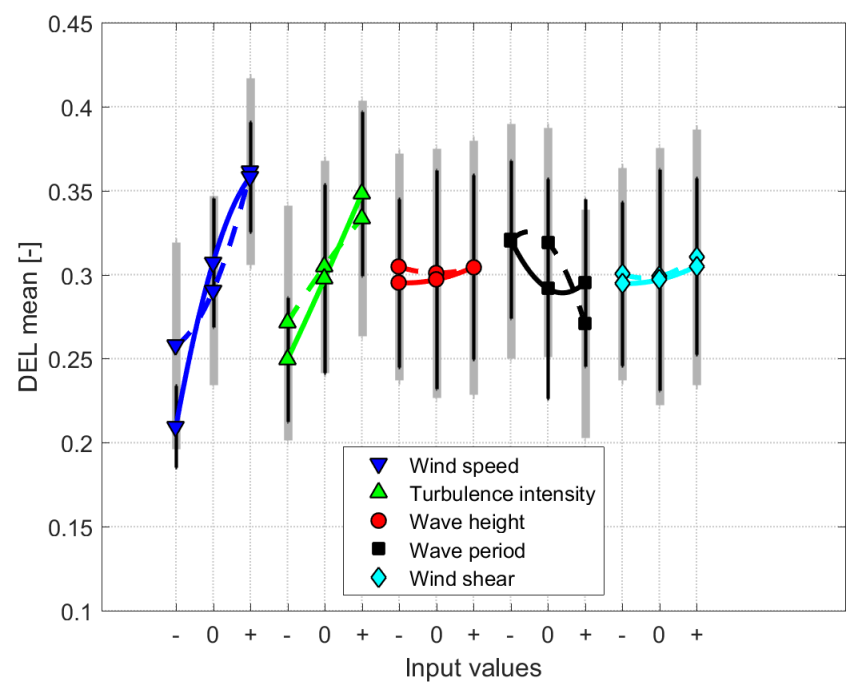

Fig. 5 Normalized fore-aft tower bending moment DEL sensitivity in partial load range $\left(v<v_{\text {rated }}\right)$ with errorbar size equivalent to $1 \sigma$ for full factorial (grey vertical) and Box-Behnken (black vertical) 


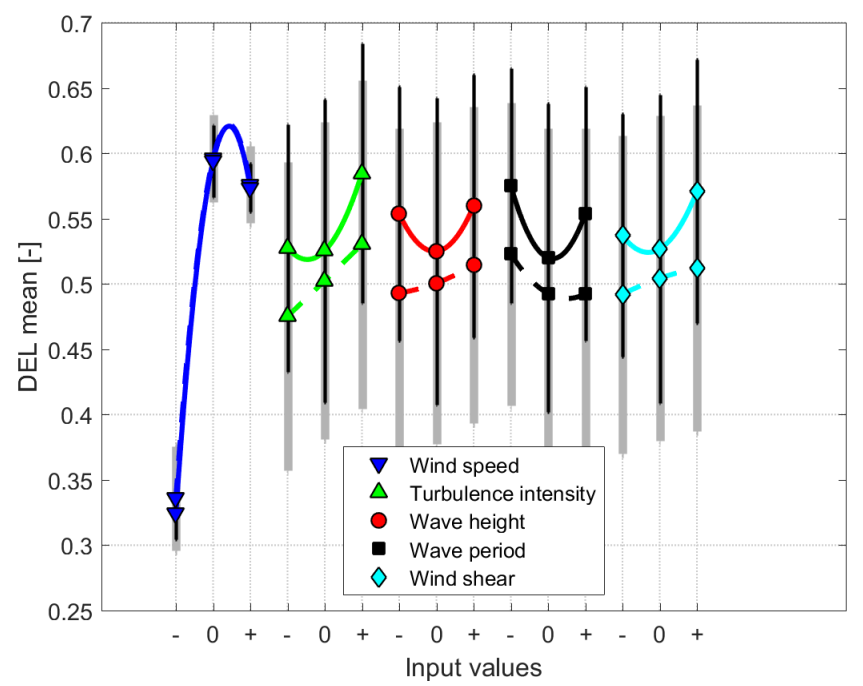

Fig. 6 Normalized fore-aft tower bending moment DEL sensitivity in transition load range $\left(v \sim v_{\text {rated }}\right)$ with errorbar size equivalent to $1 \sigma$ for full factorial (grey vertical) and Box-Behnken (black vertical)

calculated for the loads at the different factor levels ("-", "0", and "+") (see Table 1). In particular, this means, for example, the calculation of the mean value and standard deviation from all simulations with the setting of "-“, "0", and "+" for $v_{\text {hub }}$ (see Fig. 5). This procedure can also be used in the same way to highlight the interaction effects between environmental parameters. The main effects of the considered coefficients on the tower base fore-aft bending moment are presented for the three different load ranges in Fig. 5, Fig. 6, and Fig. 7, respectively, which highlight the overall importance of wind speed and turbulence intensity over other parameters.

On the basis of the evaluation of the main effects of the tower base fore-aft bending moment, the Box-Behnken method provides a good estimate of the influence of the wind speed in the transition and full load ranges. In the partial load range, the influence of the wind speed is not correctly reproduced by the Box-Behnken

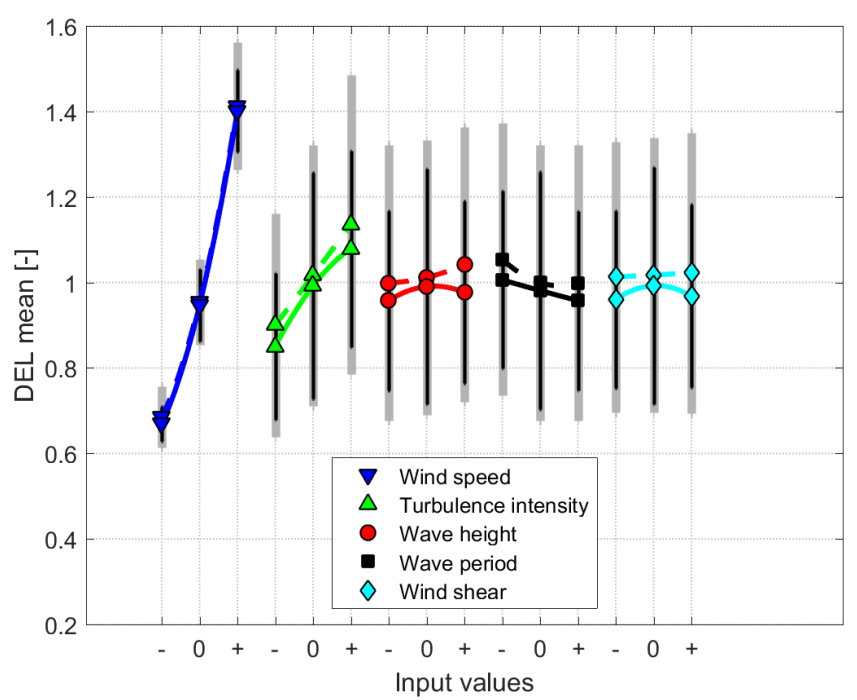

Fig. 7 Normalized fore-aft tower bending moment DEL sensitivity in full load range $\left(v>v_{\text {rated }}\right)$ with errorbar size equivalent to $1 \sigma$ for full factorial (grey vertical) and Box-Behnken (black vertical) design. Also, deviations of up to $10 \%$ between the Box-Behnken and full factorial designs are present for the sensitivity parameters of turbulence intensity, wave height, wave period, and wind shear. In the transition load range, there is some visible overestimation of the influence of the turbulence intensity, wave height, wave period, and wind shear, which turns into slight underestimation in the full load range. The lower performance of the Box-Behnken design in the partial load range with respect to the wind speed can be attributed to the selection of the design points and the resulting quality of the response surface. The design point selection leads to a lower density of results towards the edges of the hypercube, and hence the quality of the response surface decreases at the outer points of the hypercube. In the partial load range, this affects the wind speed more than in other load ranges as here the relative influence of the other environmental parameters is larger.

\section{Fatigue Load Sensitivity Analysis of Further Positions of the Wind Turbine and Substructure}

Figure 8 summarizes the linear regression coefficients $b_{i}$ for the response surfaces at different positions of the turbine and substructure. It can be seen that the influence of the wind speed is strongly predominant on the turbine and the upper parts of the substructure. The growing influence of the marine environment closer to the structural parts near the mudline and brace can also be seen. However, in the full load range, the wind speed remains a predominant factor for all evaluated positions. Furthermore, the exceptionally large influence of the wind shear on the blade loads around the rated wind speed (TLR) is visible.

\section{Evaluation of Box-Behnken Method for Selection of Design Points}

A more detailed investigation of the Box-Behnken method is possible through an analysis of the resulting response surface that can be obtained through the application of Eqs. 2 to 5. Once the

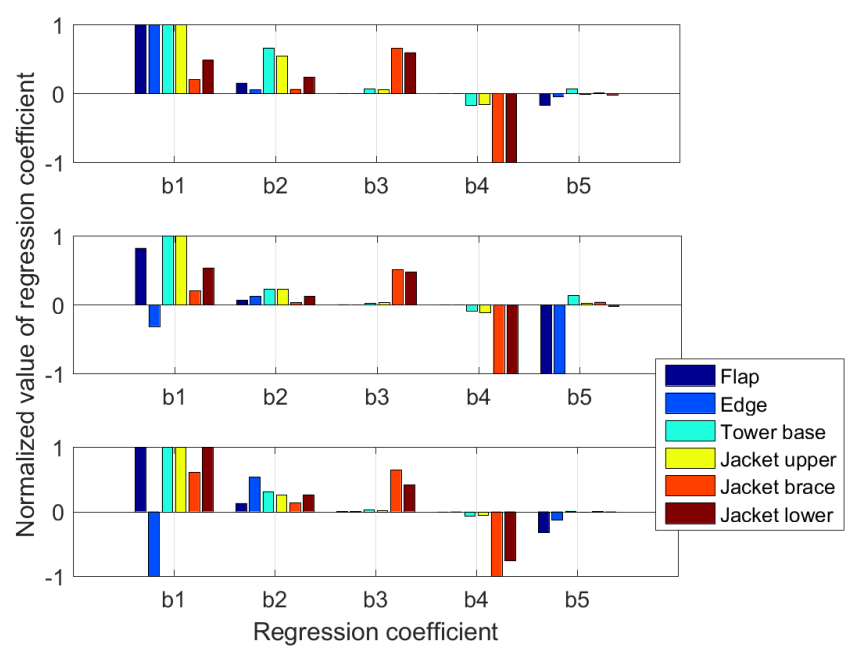

Fig. 8 Normalized linear regression coefficients for different load ranges from Box-Behnken design results. Considered positions are flapwise blade root bending moment, edgewise blade root bending moment, tower base fore-aft bending moment, upper jacket leg strain, jacket brace strain (close to mudline node), and lower jacket leg strain. Relation of coefficients to environmental conditions is as follows: $b_{1}$ is related to wind speed, $b_{2}$ is related to turbulence intensity, $b_{3}$ is related to wave height, $b_{4}$ is related to wave period, and $b_{5}$ is related to wind shear. 


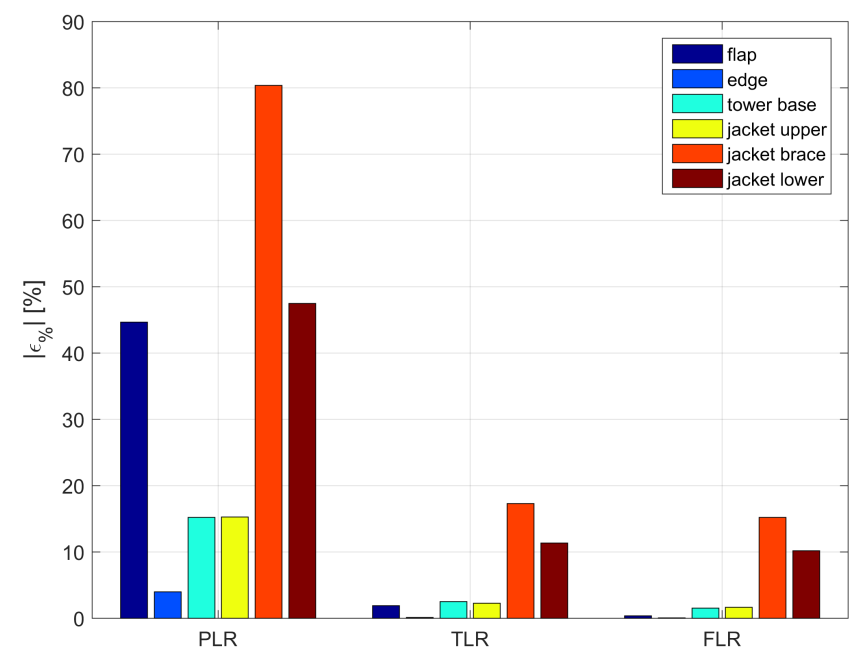

Fig. 9 Evaluation of the Box-Behnken method for the selection of design points for the fatigue load simulations based on normalized absolute residuals $\left|\varepsilon_{\%}\right|$ (see Eq. 7)

regression coefficients are obtained, they allow a detailed sensitivity analysis of the overall structure. We present this here, focusing on the evaluation of fatigue loads.

In order to evaluate the Box-Behnken approach quantitatively, we performed a residual analysis comparing the full factorial results for DEL with the values calculated through the metamodel. For this, normalized absolute residuals were calculated:

$$
\left|\boldsymbol{\varepsilon}_{\%}\right|=\frac{\left|y_{\mathrm{reg}, B B}\left(\mathbf{x}_{\mathrm{envir}}\right)-y_{F F}\left(\mathbf{x}_{\mathrm{envir}}\right)\right|}{y_{F F}\left(\mathbf{x}_{\mathrm{envir}}\right)} \cdot 100 \%
$$

where $y_{\text {reg, } B B}\left(\mathbf{x}_{\text {envir }}\right)$ are DEL results from the regression model (Box-Behnken) and $y_{F F}\left(\mathbf{x}_{\text {envir }}\right)$ are results from the full factorial simulation study at a given combination of environmental parameters $\mathbf{x}_{\text {envir }}$. The results of the analysis were categorized and summarized, as shown in Fig. 9.

Overall, compared to the full factorial analysis, the BoxBehnken method provides a good estimation for load ranges and positions that are mostly influenced by aerodynamic loads. The results show that for comparably small DEL values (PLR) and for values at positions close to the mudline, the regression based on the Box-Behnken design achieves lower-quality results. The greater error at small DEL values can be partially related to the nature of the relative comparison. For smaller values, the same absolute error results in a greater relative error. It should be noted, however, that the influence of the wind speed on the DEL is also misinterpreted when the Box-Behnken model is applied (see Fig. 5). The applicability of the Box-Behnken design at low wind speeds should be further investigated in the future. The lower performance of the Box-Behnken design at positions close to the mudline could be due to the influence of excitation close to the natural frequencies of the overall structure that cannot be adequately interpolated by the Box-Behnken meta-model. As a general rule, the residuals due to the application of the Box-Behnken design seem to be conservative.

\section{Validation of Fatigue Load Variation}

Figure 10 gives a qualitative comparison between measurements, showing the results from the full factorial design and the Box-Behnken design. For further comparison, the results of an independent simulation study focusing on IEC 61400-3 DLC 1.2 simulations are provided (IEC TS 61400-3, 2009). There, 100

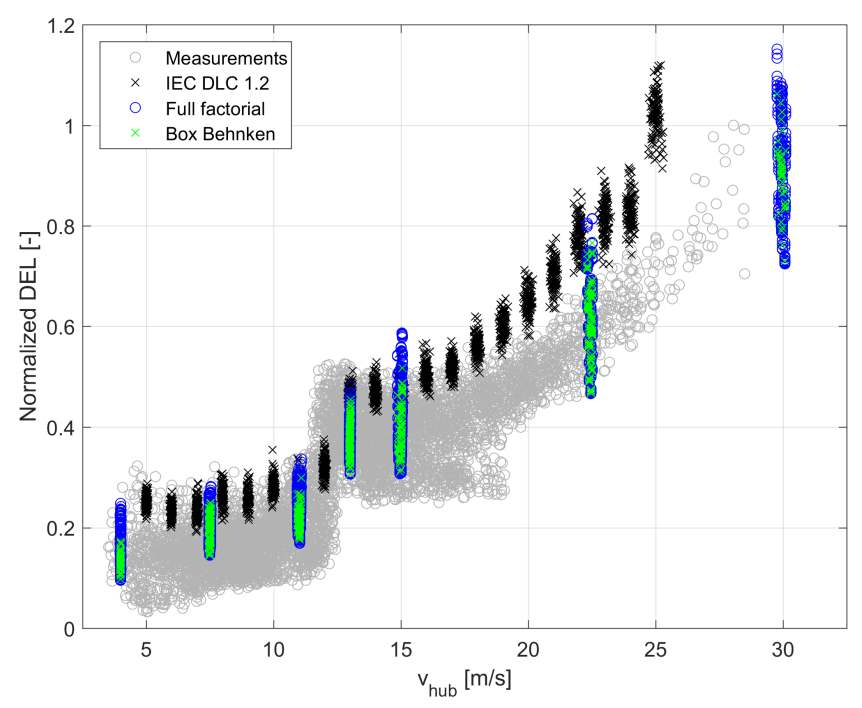

Fig. 10 Validation of full factorial, Box-Behnken, and IEC 61400-3 DLC 1.2 simulation results against selected measurement data. Evaluated data for tower base fore-aft bending moment.

simulations are performed per considered wind bin, and for the turbulence intensity as the second-most influential parameter, the 90th percentile value from the distribution of the turbulence intensity is used (Müller and Cheng, 2016). These results show that the load variations are limited, and the environmental parameters are in general conservatively chosen from guideline environmental assumptions. Regarding the results from the simulation study presented here, the general offset of all simulations in the partial load range is deemed to be linked to the distance of the FINO1 met mast from the turbine and the interaction of the turbine with the flow surrounding it. This means that the flow field obtained from FINO1 measurements, which is used as simulation input, is not the same as the one that is present in front of the turbine. Close to the rated wind speed, the spread of the loads provided by the simulation study is not as large as that from the measurements. This could originate from the adverse interaction of the controller with the real environment, e.g., the controller cannot deal with the turbulence level and aerodynamic damping is not captured correctly, which have been previously observed to result in increased loads.

Overall, the inclusion of the variance of environmental parameters through, for example, the use of the design of experimental methods captures well the variation of fatigue loads introduced by the variation of environmental conditions and is better than the simulations carried out under the environmental conditions described in the guideline. A direct quantitative comparison of the occurrence possibility of the different load values is necessary for further investigation of the implications for lifetime damage. However, this needs to link the probability of the environmental parameters to the derived Box-Behnken meta-model, which is beyond the scope of the presented work.

\section{EVALUATION OF EXTREME LOAD SIMULATION STUDY AND VALIDATION OF MAXIMUM LOADS}

The objective of the second part of the presented work is to validate the measured extreme loads. Here, the maximum loads from a ten-minute time series measured during power production are used. This represents DLC 1.1 in the IEC 61400-3 (IEC TS 61400-3, 2009). The goal of the evaluation is to reproduce the measured maximum loads with maximum loads obtained in 


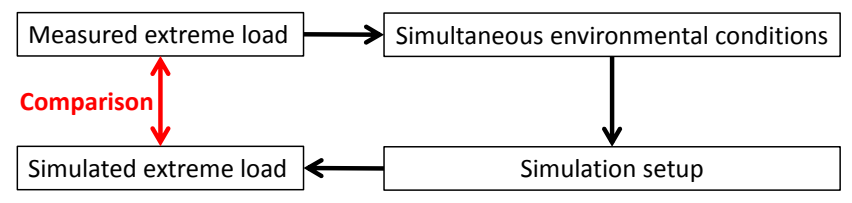

Fig. 11 Applied workflow for validation of extreme loads

simulations through the application of the same mean environmental data to the simulation model (see Fig. 11).

It is expected that adequate representation of the effects that lead to extreme loading requires high-resolution environmental data. However, even though $1-\mathrm{Hz}$ data is measured for wind speed, for example, the measurements currently available within the project do not provide the necessary spatial resolution of the environmental parameters to allow an extreme load validation in the time domain. Regarding spatial resolution, the wind speed is measured at one anemometer on the FINO1 platform that is positioned $400 \mathrm{~m}$ away from the considered turbine. A gust passing by the platform is likely to arrive at the turbine with a delay of almost a minute. Additionally, since the measurement is performed at a single point in space, it is likely that a gust heading towards the turbine is not even registered by the measurement devices. The area of the rotor is too large to accurately extrapolate the high-resolution wind speed information from a single point measurement to all evaluation points of the rotor domain. Thus, the high-resolution time series does not provide sufficient insight into the loads occurring at the turbine, and hence a time series validation based on a single point measurement $400 \mathrm{~m}$ away from the turbine was not considered.

Because the aforementioned effects cannot be captured by the high-resolution time series, the use of ten-minute averages and statistics from the environmental data recommended by guidelines for load analysis is regarded as a realistic possibility to reproduce transient extreme loads. It can also be shown that extreme environmental conditions are not necessarily responsible for extreme loads during power production, i.e., the largest maximum loads are often measured around the rated wind speed.

The question thus remains if extreme loads can be reproduced by the application of mean environmental conditions and added stochastic variance. Therefore, specific events are selected, and the measured environmental data are fed into the simulation model. The resulting calculated extreme loads are then compared to the measured loads.

In order to show that the simulation model and common design procedures are generally capable of capturing extreme loads measured during power production, the maximum loads from the results of the aforementioned simulation studies (DOE, IEC) are compared to measured extreme loads.

\section{Environmental Conditions for Extreme Load Simulations}

For the evaluation of extreme loads, the same measurement database is used that is used for the fatigue load evaluation. In order to obtain simulation input for extreme load validation, maximum load measurements above the $98^{\text {th }}$ percentile are selected. From this subset only those events are selected when measurements of the environmental parameters are available. The values for $v_{\text {hub }}, T i, H_{s}, T_{p}, \alpha$, wind direction, and wave direction are considered. The resulting set of mean environmental conditions is presented in Table 2. The small number of combinations that allows an actual analysis of the extreme loads highlights the difficulty of a thorough validation procedure at full scale when unlikely events are considered such as fifteen ten-minute time series in a period of ten months.

\begin{tabular}{rcccccccr}
\hline & $\begin{array}{c}v \\
\text { \# } / \mathrm{s}]\end{array}$ & $\begin{array}{c}T i \\
{[-]}\end{array}$ & $\begin{array}{c}H_{s} \\
{[\mathrm{~m}]}\end{array}$ & $\begin{array}{c}T_{p} \\
{[\mathrm{~s}]}\end{array}$ & $\begin{array}{c}\alpha \\
{[-]}\end{array}$ & $\begin{array}{c}\text { Wind } \\
\left.\text { dir. [ }{ }^{\circ}\right]\end{array}$ & $\begin{array}{c}\text { Wave } \\
\left.\text { dir. [ }{ }^{\circ}\right]\end{array}$ & $\begin{array}{r}\text { Dev. } \\
{[\%]}\end{array}$ \\
\hline 1 & 11.7 & 0.052 & 1.3 & 5.7 & 0.14 & 254 & 281 & 6.49 \\
2 & 12.0 & 0.024 & 1.3 & 8.6 & 0.14 & 269 & 338 & 16.78 \\
3 & 12.1 & 0.048 & 2.8 & 10.6 & 0.36 & 219 & 337 & -2.69 \\
4 & 12.2 & 0.119 & 4.0 & 13.0 & 0.11 & 241 & 331 & -9.68 \\
5 & 12.2 & 0.052 & 1.2 & 7.7 & 0.16 & 268 & 332 & 14.54 \\
6 & 12.4 & 0.026 & 1.5 & 7.2 & 0.09 & 228 & 286 & -2.29 \\
7 & 12.5 & 0.018 & 1.6 & 7.3 & 0.13 & 229 & 286 & -1.20 \\
8 & 12.5 & 0.034 & 1.1 & 5.2 & 0.05 & 248 & 277 & 3.04 \\
9 & 12.5 & 0.037 & 0.7 & 3.4 & 0.14 & 224 & 252 & -2.18 \\
10 & 12.5 & 0.143 & 2.0 & 7.0 & 0.14 & 210 & 260 & 3.29 \\
11 & 12.6 & 0.029 & 1.3 & 7.1 & 0.05 & 263 & 335 & 14.47 \\
12 & 13.0 & 0.039 & 1.1 & 5.3 & 0.26 & 262 & 303 & 9.12 \\
13 & 13.0 & 0.032 & 1.7 & 5.5 & 0.02 & 253 & 264 & 7.09 \\
14 & 13.6 & 0.045 & 3.3 & 9.1 & 0.13 & 246 & 289 & 3.25 \\
15 & 13.9 & 0.066 & 3.0 & 7.7 & 0.07 & 261 & 261 & 14.16 \\
\hline
\end{tabular}

Table 2 Measured environmental conditions during occurrence of above 98th percentile loads. Relative deviation in percent is given for simulation results (maximum of six seeds) with respect to measured loads. A positive deviation means that measured loads are larger than simulated loads.

\section{Overall Evaluation of Results of Simulated Extreme Loads}

For an overall evaluation of simulated extreme loads, results from the previously mentioned IEC load simulation study (100 simulations per considered bin) and the full factorial and BoxBehnken simulation study (maximum loads) are compared with the measurement data (see Fig. 12). This evaluation shows that overall, the simulation model is able to capture the variation of extreme loads experienced in the field, with some conservatism implied (DOE study), especially for wind speeds below rated. Compared to this, IEC simulations show less variation and higher conservatism, especially for wind speeds above rated. Around the rated wind speed where the largest extreme loads during power production occur, the variation of IEC loads is very small compared to the variation of both the measured and DOE-based loads (see Fig. 13).

The overall large variation of IEC-based loads, which is almost comparable to that of the DOE results, indicates a strong influ-

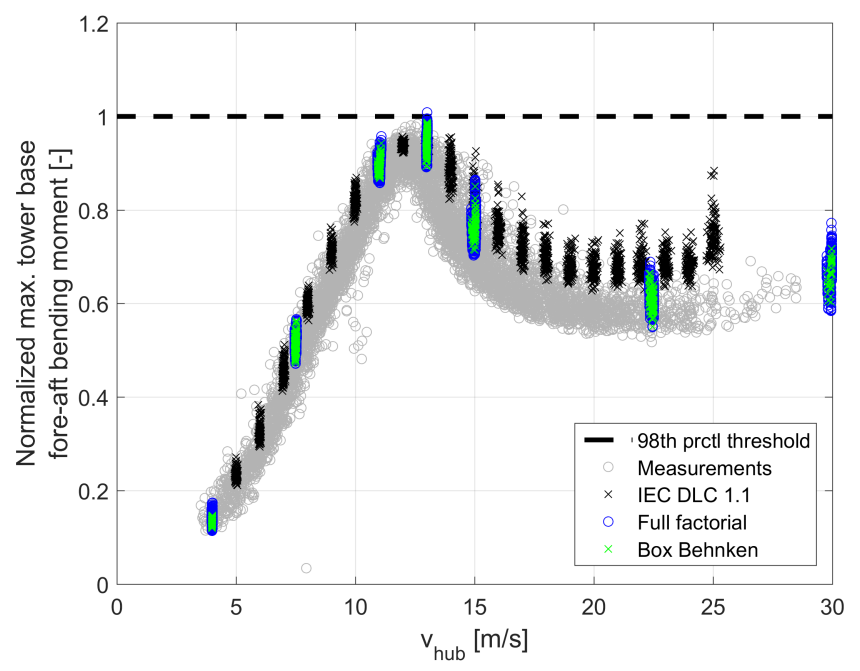

Fig. 12 Measured and simulated (IEC DLC 1.1 and DOE) maximum tower base fore-aft bending moments 


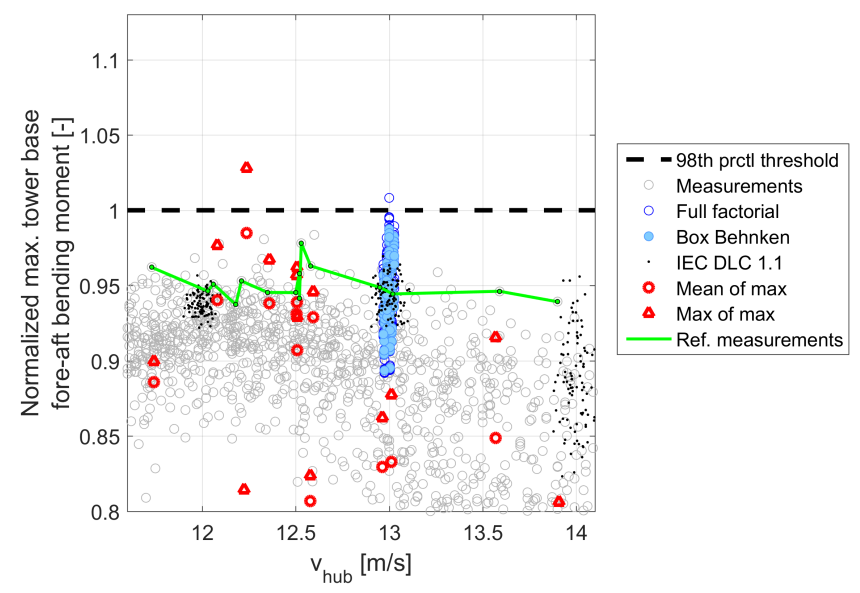

Fig. 13 Detailed view of measured and simulated maximum tower base fore-aft bending moments around rated wind speed

ence of turbulence intensity on extreme loads at the tower base below and above the rated wind speed. This variation also underlines the necessity of using a sufficiently large amount of seeds for load extrapolation. At the rated wind speed, however, the load variation is small compared to that of the DOE results and measurements, and hence it is recommended to consider a wider range of environmental conditions in the transition region.

\section{Simulation Setup for Validation of Extreme Loads}

For each of the environmental conditions provided in Table 2, six seeds with different wind input files are simulated. The values of the maximum tower base fore-aft bending moment are then collected. For each of the fifteen established environmental conditions, the mean of the maxima and the maximum of the maxima are then plotted against the reference measurement data for visual comparison (see Fig. 13).

\section{Evaluation of Results from Reproduction of Largest Extreme Loads}

Regarding the reproduction of extreme loads, the simulated maximum values generally fit the scattered distribution of maximum loads from the measurements. However, a considerable amount of the results stays below the implemented threshold of the $98^{\text {th }}$ maximum load percentile. Regarding the maximum values of the six maximum load results for each simulated event, deviations of up to $16.7 \%$ are observed.

Through the application of trend lines, a link between the deviations and environmental conditions can be established. Here, slight dependencies of the deviations can be observed for the peak period (quadratic trend coefficient of determination $R^{2}=$ 0.51 ), wind direction (linear trend $R^{2}=0.67$ ), and wave direction (quadratic trend $R^{2}=0.43$ ).

The results show that mean environmental conditions that are present when extreme loads occur generally cannot be used to determine extreme loads on the turbine through simulation. This means that the validation procedure, which has been previously applied to fatigue loads, i.e., to the comparison of loads from specific environmental events, is not applicable to extreme loads when environmental, ten-minute averages are considered. Transient events that are not captured under the mean environmental conditions are deemed to be responsible for the occurrence of extreme loads.

Another aspect, which is not addressed here, is the influence of the behavior of the controller on measured extreme loads. As the largest maximum loads occur around the rated wind speed, activity in the pitch system could play an important factor that may not be adequately reproduced in the simulation environment. In order to obtain a high-resolution representation of the wind environment in front of the turbine, a rotor-effective wind speed (Soltani et al., 2013) could replace the measurements from the FINO1 anemometer used in this work.

\section{SUMMARY, CONCLUSIONS, AND OUTLOOK}

The presented work focused on the validation of load assumptions for both fatigue and ultimate loads. Two simulation designs (full factorial and Box-Behnken) were presented to show the possibility of correctly including the range of environmental loads in the state-of-the-art simulation tools. A basis was provided for the validation and verification of the environmental assumptions for fatigue loads in the certification process. Additionally, the potential of the applied designs of experiments for fatigue load sensitivity study was evaluated. Good agreement of the meta-model derived through the Box-Behnken approach was established, with special care to be taken at low wind speeds and locations within the turbine that are sensitive to periodic excitation from wave loads (e.g., braces) or self-excitation (e.g., tower side-side bending moment). Here it was shown how the tower base moments and even locations at the upper jacket were little influenced by the marine environment. Also, a strongly increased influence of the wind shear on blade loads around the rated wind speed could be observed.

The applied validation of the largest maximum loads for the tower base fore-aft bending moment based on mean environmental data showed no reliable indication of applicability. As a general statement, it was noted that the validation of extreme loads requires high-resolution input of the environmental data as extreme loads cannot be reproduced reliably by the application of a simplified stochastic representation of the environment.

Overall, the findings of the presented work can be summarized as follows:

- State-of-the-art integrated simulation models are capable of predicting the variation of both fatigue and extreme loads when the variation of environmental conditions is considered.

- The application of DOE methods for the determination of the fatigue load range is reasonable for the transition and full load ranges when the aerodynamic loads are dominating. Special care needs to be taken for locations within the turbine that are highly influenced by periodic excitation.

- The largest extreme loads cannot be reproduced by tenminute average loads and stochastic models included in available tools. Thus, a comparison based on time series measurements is necessary for extreme load validation.

- The variation of extreme loads based on IEC calculations around the rated wind speed is lower than that based on simulations that take into account the thorough variation of environmental parameters. Thus, including the variation of environmental conditions in the determination of extreme loads could be interesting.

Upcoming research will focus on the inclusion of the probability of discrete environmental events that will enable a quantitative comparison between certification simulations and DOE-based simulations and measurements. Also, the inclusion of the rotoreffective wind speed for the simplified time series validation of extreme loads will be addressed.

\section{ACKNOWLEDGEMENTS}

This research is part of the RAVE projects OWEA "Verification of Offshore Wind Turbines" and OWEA LOADS. It is funded by 
the Federal Ministry for Economic Affairs and Energy (BMWI). The authors thank Senvion GmbH for its support and the possibility of validating the Flex5-Poseidon model through the use of measurement data.

\section{REFERENCES}

Böker, C (2010). Load Simulations and Local Dynamics of Support Structures for Offshore Wind Turbines, Shaker Verlag, 192 pp.

Box, GEP, and Behnken, DW (1960). "Some New Three Level Designs for the Study of Quantitative Variables," Technometrics, 2(4), 455-475.

http://dx.doi.org/10.1080/00401706.1960.10489912.

DOTI (2015). Deutsche Offshore-Testfeld-und Infrastruktur $\mathrm{GmbH} \&$ Co. KG. Retrieved from www.alpha-ventus.de. Accessed on 14 December 2015.

FINO-Offshore (2015). Forschungs-und Entwicklungszentrum Fachhochschule Kiel GmbH. Retrieved from http://www.finooffshore.de/. Accessed on 14 December 2015.

Gundlach, C (2004). Entwicklung eines Ganzheitlichen Vorgehensmodells zur Problemorientierten Anwendung der Statistischen Versuchsplanung, PhD Dissertation, Kassel University Press, Germany.

IEC TS 61400-3 (2009). Wind Turbines - Part 3: Design Requirements for Offshore Wind Turbines, Edition 1.0, International Electrotechnical Commission.

IEC TS 61400-13 (2001). Wind Turbine Generator Systems Part 13: Measurement of Mechanical Loads, International Electrotechnical Commission.

Jonkman, J, and Musial, W (2010). Offshore Code Comparison Collaboration (OC3) for IEA Task 23 Offshore Wind Technology and Deployment, NREL/TP-5000-48191, National Renewable Energy Laboratory, Golden, CO, USA.

Kaufer, D, and Cheng, PW (2014). "Validation of an Integrated Simulation Method with High-Resolution Load Measurements of the Offshore Wind Turbine Repower 5M at Alpha Ventus," J Ocean Wind Energy, ISOPE, 1(1), 30-40.

Kleijnen, JPC (2008). Design and Analysis of Simulation Experiments, Springer Science + Business Media, 239 pp.

Koukoura, C, Natarajan, A, Krogh, T, and Kristensen, OJ (2013). "Offshore Wind Turbine Foundation Model Validation with Wind Farm Measurements and Uncertainty Quantification," Proc 23rd Int Offshore Polar Eng Conf, Anchorage, AK, USA, ISOPE, 1, 119-125.

Montgomery, DC (2013). Design and Analysis of Experiments, John Wiley, $730 \mathrm{pp}$.

Müller, K, and Cheng, PWC (2016). "Validation of Uncertainty in IEC Damage Calculations Based on Measurements from Alpha Ventus," Energy Procedia (under review).

Müller, K, et al. (2014). "Improved Tank Test Procedures for Scaled Floating Offshore Wind Turbines," Proc Int Wind Eng Conf, Hannover, Germany.

http://elib.uni-stuttgart.de/handle/11682/8278.
Niesłony, A (2009). "Determination of Fragments of Multiaxial Service Loading Strongly Influencing the Fatigue of Machine Components," Mech Syst Sig Process, 23(8), 2712-2721.

http://dx.doi.org/10.1016/j.ymssp.2009.05.010.

OWEA Loads (2015). Fraunhofer IWES. Retrieved from http:// rave.iwes.fraunhofer.de/rave/pages/raveLoads. Accessed on 14 December 2015.

Owec Tower AS (2015). Owec Tower AS. Retrieved from http:// www.owectower.no/our-product/. Accessed on 14 December 2015.

Øye, S (1999). FLEX5 User Manual, Danske Techniske Hogskole, Denmark.

Popko, W, et al. (2012). "Offshore Code Comparison Collaboration Continuation (OC4), Phase I - Results of Coupled Simulations of an Offshore Wind Turbine with Jacket Support Structure," Proc 22nd Int Offshore Polar Eng Conf, Rhodes, Greece, ISOPE, 1, 337-346.

RAVE (2015). Research at Alpha Ventus, Fraunhofer IWES. Retrieved from www.rave-offshore.de. Accessed on 14 December 2015 .

Söker, H, Illig, C, Cosack, N, Kröning, J, and Damaschke, M (2006). "A Guide to Design Load Validation," Deutsche Windenergie-Konferenz (DEWEK), Bremen, Germany. Available from http://www.dewi.de/dewi/fileadmin/pdf/publications/ Publikations/14_2_Soeker.pdf.

Soltani, M, et al. (2013). "Estimation of Rotor Effective Wind Speed: A Comparison," IEEE Trans Control Syst Technol, 21(4), 1155-1167. http://dx.doi.org/10.1109/TCST.2013.2260751.

Türk, M (2008). Ermittlung Designrelevanter Belastungsparameter für Offshore-Windkraftanlagen, $\mathrm{PhD}$ Dissertation, Universität Köln, Germany.

Türk, M, and Emeis, S (2010). "The Dependence of Offshore Turbulence Intensity on Wind Speed," J Wind Eng Ind Aerodyn, 98(8-9), 466-471. http://dx.doi.org/10.1016/j.jweia.2010.02.005.

Westerhellweg, A, Riedel, V, and Neumann, T (2011). "Comparison of LIDAR and UAM-based Offshore Mast Corrections," Proc EWEA 2011, Brussels, Belgium. Available from http://www.dewi.de/dewi_res/fileadmin/pdf/publications/ Publikations/PO.087_EWEA2011paper.pdf.

Yde, A, Larsen, TJ, Hansen, AM, Fernandez, M, and Bellew, S (2015). "Comparison of Simulations and Offshore Measurement Data of a Combined Floating Wind and Wave Energy Demonstration Platform," J Ocean Wind Energy, ISOPE, 2(3), 129-137. http://dx.doi.org/10.17736/jowe.2015.jcr34.

Zierath, J, Rachholz, R, Woernle, C, and Müller, A (2014). "Load Calculation on Wind Turbines: Validation of Flex5, Alaska/ Wind, MSC. Adams and SIMPACK by Means of Field Tests," Proc ASME 2014 Int Des Eng Tech Conf Comput Inf Eng Conf, Buffalo, NY, USA, ASME, V007T05A009. http://dx.doi.org/10.1115/DETC2014-34670. 